\title{
Fusion of Decisions Transmitted Over Rayleigh Fading Channels in Wireless Sensor Networks
}

\author{
Ruixin Niu, Member, IEEE, Biao Chen, Member, IEEE, and Pramod K. Varshney, Fellow, IEEE
}

\begin{abstract}
In this paper, we revisit the problem of fusing decisions transmitted over fading channels in a wireless sensor network. Previous development relies on instantaneous channel state information (CSI). However, acquiring channel information may be too costly for resource constrained sensor networks. In this paper, we propose a new likelihood ratio (LR)-based fusion rule which requires only the knowledge of channel statistics instead of instantaneous CSI. Based on the assumption that all the sensors have the same detection performance and the same channel signal-to-noise ratio (SNR), we show that when the channel SNR is low, this fusion rule reduces to a statistic in the form of an equal gain combiner (EGC), which explains why EGC is a very good choice with low or medium SNR; at high-channel SNR, it is equivalent to the Chair-Varshney fusion rule. Performance evaluation shows that the new fusion rule exhibits only slight performance degradation compared with the optimal LR-based fusion rule using instantaneous CSI.
\end{abstract}

Index Terms-Decision fusion, diversity combining, fading channels, wireless sensor networks.

\section{INTRODUCTION}

W ITH the significant advances in the fields of networking, wireless communications, microfabrication and microprocessors, wireless sensor networking has become a fast-growing research area. A typical wireless sensor network (WSN) is made up of a large number of small, inexpensive, and low-power sensors, which are deployed in the environment to collect observations. Each sensor node preprocesses and extracts information from the raw observations. Each sensor node also has the ability to communicate with other sensor nodes or a central node (fusion center) via wireless channels. Usually, there is a fusion center that jointly processes data from local sensors and forms a global and more precise situational assessment. Because sensors are connected via a wireless communication network, WSNs can be deployed rapidly and in a flexible manner. This flexibility, along with the enhanced surveillance coverage and sensing performance, makes WSNs particularly appealing for a number of applications, such as battlefield surveillance, security, monitoring of traffic and environment.

\footnotetext{
Manuscript received May 19, 2004; revised April 4, 2005. This work was presented in part at CISS'03, Baltimore, MD, March 2003. This work was supported in part by the National Science Foundation under Grant ECS-0501534. The associate editor coordinating the review of this manuscript and approving it for publication was Prof. Yucel Altunbasak.

The authors are with Department of Electrical Engineering and Computer Science, Syracuse University, Syracuse, NY 13244 USA (e-mail: rniu@ecs.syr.edu; bichen@ecs.syr.edu; varshney@ecs.syr.edu).

Digital Object Identifier 10.1109/TSP.2005.863033
}

Many aspects of WSNs have been investigated recently, such as efficient routing protocols [1], distributed data compression and transmission, and collaborative signal processing [2]. We are particularly interested in the information processing task at the fusion center, and more specifically, in the decision fusion task. For a distributed detection system, the conventional wisdom is to consider communication and decision fusion as independent entities and design these two functions separately. For systems employing high signal-to-noise ratio (SNR) and/or effective channel error correction coding, communication may have extremely low error rates and can be assumed lossless, meaning that the local decisions can be transmitted to the fusion center without errors. Based on this assumption, numerous results on the classical distributed detection (decision fusion) problem have been presented in the literature. In [3] and [4], optimum fusion rules have been obtained under the conditional independence assumption. Decision fusion with correlated observations has been studied in [5]-[8]. There also exist many papers dedicated to the problem of distributed detection with constrained system resources [9]-[15]. Specifically, these papers have proposed solutions to optimal bit allocation (or sensor selection) given a constraint on the total amount of communications. While many of the obtained results are quite enlightening, the lossless communication assumption, however, should be subject to careful scrutiny. Increasing power and/or employing powerful error correction codes may not always be possible as most WSN are resource constrained (in both energy and bandwidth). Furthermore, in a hostile environment, the power of transmitted signal should be kept to a minimum to attain a low probability of intercept/detection (LPI/LPD). Therefore, it may be necessary in many situations to tolerate the loss during data transmission to some extent. To overcome this loss, it is highly desirable to integrate the communication and decision fusion functions intelligently to achieve an acceptable system performance without spending extra system resources. This motivates the study of fusion of local decisions corrupted during the transmission process due to channel fading/noise impairment. In [16], Thomopoulos and Zhang have investigated decision fusion with nonideal channels. They derived the optimum tests and corresponding thresholds both at the fusion center and at the local sensors. Their method, however, was very complex and required global knowledge of the entire system. In addition, the binary channel assumption did not allow the full integration of transmission into the decision fusion stage.

In our previous work [17], the problem of fusing decisions transmitted over fading and noisy channels in a WSN was investigated. The system model adopted in [17], which represents the process of multiple-sensor decision fusion with 


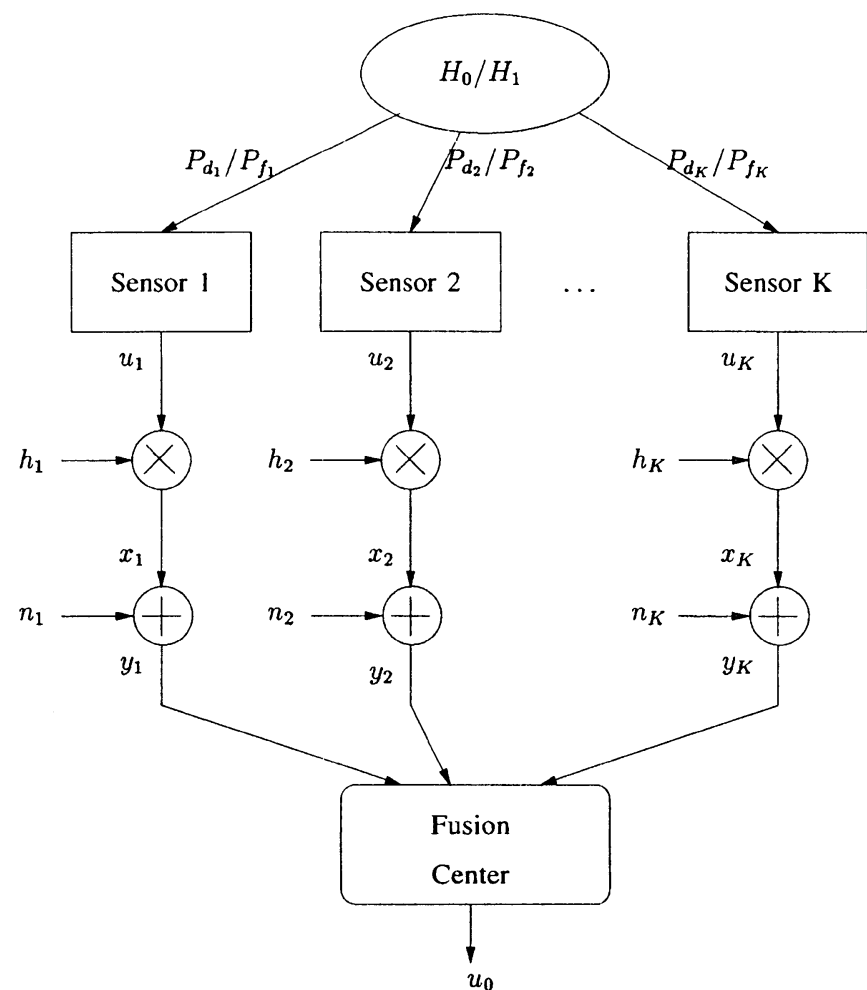

Fig. 1. Parallel fusion model in the presence of fading and noisy channels between local sensors and the fusion center. $u_{k}$ is the binary decision made by the $k$ th sensor, $h_{k}$ is the fading channel gain, $n_{k}$ is a zero-mean Gaussian random variable with variance $\sigma^{2}$, and $y_{k}$ is the observation received by the fusion center from the $k$ th sensor, where $k=1, \ldots, K$.

fading channels, is illustrated in Fig. 1. A number of sensors collect and process raw measurements, make local decisions, and transmit them over fading and noisy channels to a fusion center. The fusion center fuses the received local decisions according to a certain fusion rule and thus obtains a global decision about the presence/absence of a target. Based on this model, an optimal likelihood ratio (LR)-based fusion rule is developed in [17], which requires the maximum amount of information regarding the system, including instantaneous channel state information (CSI) and local sensors' detection performance indices. Starting from the optimal LR fusion rule, three suboptimal methods-the Chair-Varshney fusion rule, the maximum ratio combiner (MRC), and equal gain combiner (EGC) are also presented in previous work. In [17], it is shown that for low-channel SNR, MRC is near-optimal; for high SNR, the Chair-Varshney rule is near-optimal. Interestingly, the very simple EGC statistic, which requires minimum amount of information, outperforms both MRC and Chair-Varshney fusion rules for most practical SNR values.

The optimal LR-based fusion rule requires instantaneous CSI. Acquiring channel knowledge, however, may be too costly in resource constrained applications. Our goal here is to develop a new fusion rule that does not require instantaneous CSI and yet provides robust performance. In particular, we derive a new LR-based fusion rule that requires only channel statistics. This fusion rule is optimal when only local sensor performance indexes and channel statistics are available. We also show that EGC and Chair-Varshney rules are its approximations at lowand high-channel SNR, respectively. The new LR-based fusion rule consistently outperforms EGC and Chair-Varshney rules. It also has better performance than the MRC fusion rule for most practical SNR values.

In Section II, we formulate the parallel fusion problem that includes the fading channel layer and review various fusion rules developed in previous work. In Section III, we derive the new LR-based fusion rule assuming only the knowledge of fading statistics. Based on the assumption that all the sensors have the same detection performances and the same channel SNRs, we show that EGC and Chair-Varshney fusion statistics are high- and low-SNR approximations of the new LR-based fusion statistic, respectively. Performance analysis is contained in Section IV. There, closed-form results for the performance of the two stage approach using the Chair-Varshney rule are derived. Using the so-called deflection coefficient, we show that the new fusion rule is better than both EGC and Chair-Varshney rules. Simulation results are also provided to confirm our analysis. In addition, we show that the new fusion rule is robust and outperforms both EGC and Chair-Varshney fusion rules when the assumption of identical sensor detection performances and identical channel SNRs is relaxed. We present some concluding remarks in Section V.

\section{Problem Formulation and Previous Results}

\section{A. Three-Layer System Model}

The three-layer model for a distributed detection system in the presence of fading channels is illustrated in Fig. 1. There are two hypotheses, $H_{1}$ and $H_{0}$, under test. Each sensor obtains its own observations, processes them and makes a local decision. These local decisions are transmitted via fading and noisy channels to a fusion center. In a traditional distributed detection system model, all local decisions are assumed to be recovered perfectly at the fusion center. For WSNs operating in a fading environment, channel fading and noise impairment may render the received decisions at the fusion center unreliable, especially in resource constrained applications. Toward this end, a channel layer must be incorporated into our model to allow for the development of channel aware decision fusion rules that have proved to be more energy efficient [17]. The model shown in Fig. 1 is described below.

Local Sensor Layer: All $K$ local sensors collect observations generated under a specific hypothesis. In this paper, we assume that the observations are independent of each other across sensors conditioned on any hypothesis. After receiving its observation, each sensor makes a hard (binary) decision: $u_{k}=1$ is sent if $H_{1}$ is decided, and $u_{k}=-1$ is sent otherwise, where $k=1, \ldots, K$. The detection performance of each local sensor node can be characterized by its corresponding probability of false alarm and detection, denoted by $P_{f_{k}}$ and $P_{d_{k}}$, respectively, for the $k$ th sensor:

$$
\begin{aligned}
& P_{d_{k}}=p\left(u_{k}=1 \mid H_{1}\right) \\
& P_{f_{k}}=p\left(u_{k}=1 \mid H_{0}\right) .
\end{aligned}
$$

In general, these $\left(P_{f_{k}}, P_{d_{k}}\right)$ pairs need not be identical and they are functions of SNR's as well as thresholds at local sensors.

Fading Channel Layer: Decisions at local sensors, denoted by $u_{k}$ for $k=1, \ldots, K$ are transmitted over parallel channels 
that are assumed to undergo independent fading. In this paper, we assume flat fading channels between local sensors and the fusion center. This assumption is reasonable because most WSNs operate at short range (hence small delay spread) and low bit rate (long symbol duration) due to power and energy limitations. We further assume phase coherent reception, thus the effect of a fading channel is further simplified as a real scalar multiplication given that the transmitted signal is assumed to be binary [18]. This phase coherent reception can be either accomplished through limited training for stationary channels, or, at a small cost of SNR degradation, by employing differential encoding for fast fading channels which results in the same signal model. The statistics of the real scalar, denoted by $h_{k}$, is determined by the fading type. For example, for homogeneous scattering background, Rayleigh distribution best describes the envelope of a fading signal. In the development of fusion rules, the gain of the fading channel is considered as a (possibly unknown) constant during the transmission of a single local decision. We assume that the channel noise is additive white Gaussian and uncorrelated from channel to channel. For simplicity, we assume that the noise variances are identical for different channels. To summarize, each local decision $u_{k}$ is transmitted through a fading channel and the output of the channel (or input to the fusion center) for the $k$ th sensor is

$$
y_{k}=x_{k}+n_{k}=h_{k} u_{k}+n_{k}
$$

where $h_{k}$ is the fading channel gain and $n_{k}$ is a zero-mean Gaussian random variable with variance $\sigma^{2}$.

Fusion Center: Based on the received data $y_{k}$ for all $k$, the fusion center decides which hypothesis is more likely to be true. This is done by constructing and evaluating a fusion statistic using the observations $y_{k}$ as well as some system parameters, if available.

\section{B. Review of Previous Fusion Rules}

To facilitate our comparisons later, here we give a brief review of the fusion rules proposed in [17]. It is noteworthy that all of them involve the comparison of the corresponding fusion statistic with a threshold.

1) Optimal LR-Based Fusion Rule: By assuming instantaneous channel state knowledge regarding the fading channel and the local sensor performance indices, i.e., the $P_{f_{k}}$ and $P_{d_{k}}$ values, the optimal LR-based fusion rule has been derived in [17], with the fusion statistic (LR) given by

$$
\begin{aligned}
\Lambda(\mathbf{y}) & =\log \left[\frac{f\left(\mathbf{y} \mid H_{1}\right)}{f\left(\mathbf{y} \mid H_{0}\right)}\right] \\
& =\sum_{k=1}^{K} \log \left[\frac{P_{d_{k}} e^{-\frac{\left(y_{k}-h_{k}\right)^{2}}{2 \sigma^{2}}}+\left(1-P_{d_{k}}\right) e^{-\frac{\left(y_{k}+h_{k}\right)^{2}}{2 \sigma^{2}}}}{P_{f_{k}} e^{-\frac{\left(y_{k}-h_{k}\right)^{2}}{2 \sigma^{2}}}+\left(1-P_{f_{k}}\right) e^{-\frac{\left(y_{k}+h_{k}\right)^{2}}{2 \sigma^{2}}}}\right]
\end{aligned}
$$

where $\mathbf{y}=\left[y_{1}, \cdots, y_{K}\right]^{T}$ is a vector containing data received from all the $K$ sensors, and $\sigma^{2}$ is the variance of additive white Gaussian noise for all channels. This fusion rule requires both local sensor performance indexes and instantaneous CSI. Several suboptimum fusion rules that relax the requirements on $a$ priori knowledge have been also proposed in [17].
2) Chair-Varshney Fusion Rule: In [17], the following statistic, termed as the Chair-Varshney fusion statistic [3] has been shown to be a high-SNR approximation to $\Lambda$

$$
\Lambda_{1}=\sum_{\operatorname{sign}\left(y_{k}\right)=1} \log \frac{P_{d_{k}}}{P_{f_{k}}}+\sum_{\operatorname{sign}\left(y_{k}\right)=-1} \log \frac{1-P_{d_{k}}}{1-P_{f_{k}}} .
$$

$\Lambda_{1}$ does not require any knowledge regarding the channel gain but does require $P_{d_{k}}$ and $P_{f_{k}}$ for all $k$. This approach, however, suffers significant performance loss at low to moderate channel SNR.

3) MRC Fusion Rule: It has been shown in [17] that for small values of channel $\mathrm{SNR}, \Lambda$ in (3) reduces to

$$
\hat{\Lambda}_{2}=\sum_{k=1}^{K}\left(P_{d_{k}}-P_{f_{k}}\right) h_{k} y_{k}
$$

Further, if the local sensors are identical, i.e., $P_{d_{k}}=P_{d}$ and $P_{f_{k}}=P_{f}$ for all $k$ 's, then $\Lambda$ further reduces to a form analogous to a MRC [19] statistic

$$
\Lambda_{2}=\frac{1}{K} \sum_{k=1}^{K} h_{k} y_{k}
$$

$\Lambda_{2}$ in (6) does not require the knowledge of $P_{d}$ and $P_{f}$ provided $P_{d}-P_{f}>0$. Knowledge of the channel gain is, however, required.

4) EGC Fusion Rule: Motivated by the fact that $\Lambda_{2}$ resembles a MRC statistic for diversity combining, a third alternative in the form of an EGC has been proposed, which requires minimum amount of information:

$$
\Lambda_{3}=\frac{1}{K} \sum_{k=1}^{K} y_{k}
$$

Interestingly enough, this simple alternative outperforms both $\Lambda_{1}$ and $\Lambda_{2}$ for a wide range of SNR in terms of its detection performance [17].

\section{LiKelihood Ratio Test Based on Channel Statistics}

The optimal LR-based fusion rule presented in Section II-B-1) requires instantaneous CSI. However, for a WSN with very limited resources (energy and bandwidth), it is prohibitive to spend resources on estimating the channel every time a local sensor sends its decision to the fusion center. Thus, it is imperative to avoid channel estimation and conserve resources at the possible cost of relatively small performance degradation. This is the reasoning behind our exploration of new fusion rules that do not require instantaneous CSI. In many WSN scenarios, the statistics of the fading (random) channel and the additive Gaussian noise can be estimated in advance, and used as prior information. It is our goal to develop a new LR-based fusion rule with only the prior information regarding the channel statistics instead of the instantaneous CSI.

Assuming a Rayleigh fading channel with unit power (i.e., $E\left[h_{k}^{2}\right]=1$ ), the pdf of $h_{k}$ is:

$$
f\left(h_{k}\right)=2 h_{k} e^{-h_{k}^{2}}, \quad h_{k} \geq 0 .
$$


From (8) and using the fact that $x_{k}=u_{k} h_{k}$, it is easy to obtain

$$
f\left(x_{k} \mid u_{k}\right)=2 u_{k} x_{k} e^{-x_{k}^{2}} U\left(u_{k} x_{k}\right)
$$

where $U(\cdot)$ is a step function defined as follows:

$$
U(x)= \begin{cases}1 & x \geq 0 \\ 0 & x<0\end{cases}
$$

Further, we have the following result:

$$
\begin{aligned}
f\left(y_{k} \mid u_{k}\right) & =\frac{\int p\left(u_{k}\right) f\left(x_{k} \mid u_{k}\right) f\left(y_{k} \mid x_{k}, u_{k}\right) d x_{k}}{p\left(u_{k}\right)} \\
& =\int f\left(x_{k} \mid u_{k}\right) f\left(y_{k} \mid x_{k}\right) d x_{k}
\end{aligned}
$$

where the identity $f\left(y_{k} \mid x_{k}, u_{k}\right)=f\left(y_{k} \mid x_{k}\right)$, which is obtained due to the fact that $u_{k}, x_{k}$, and $y_{k}$ form a Markov chain, has been used. Combined with the fact that $n_{k}$ is a zero-mean Gaussian random variable with variance $\sigma^{2}$, we have Lemma 1 .

Lemma 1: The conditional pdf of $y_{k}$, the observation from sensor $k$, given local decision $u_{k}$ is

$$
\begin{aligned}
f\left(y_{k} \mid u_{k}\right)=\frac{2 \sigma}{\sqrt{2 \pi}\left(1+2 \sigma^{2}\right)} e^{-\frac{y_{k}^{2}}{2 \sigma^{2}}} & \\
\times & {\left[1+u_{k} \sqrt{2 \pi} a y_{k} e^{\frac{\left(a y_{k}\right)^{2}}{2}} Q\left(-a u_{k} y_{k}\right)\right] }
\end{aligned}
$$

where $a=1 /\left(\sigma \sqrt{1+2 \sigma^{2}}\right)$, and $Q(\cdot)$ is the complementary distribution function of the standard Gaussian, i.e.,

$$
Q(x)=\int_{x}^{\infty} \frac{1}{\sqrt{2 \pi}} e^{-\frac{t^{2}}{2}} d t .
$$

Proof: See Appendix I.

Once we have $f\left(y_{k} \mid u_{k}\right)$, it is easy to obtain $f\left(y_{k} \mid H_{1}\right)$, $f\left(y_{k} \mid H_{0}\right)$ and hence the likelihood ratio, as stated in the following theorem.

Theorem 1: The log-likelihood ratio based on the knowledge of channel statistics and local detection performance indexes is

$$
\begin{aligned}
\Lambda_{4} & =\log \left[\frac{f\left(\mathbf{y} \mid H_{1}\right)}{f\left(\mathbf{y} \mid H_{0}\right)}\right] \\
& =\sum_{k=1}^{K} \log \left\{\frac{1+\left[P_{d_{k}}-Q\left(a y_{k}\right)\right] \sqrt{2 \pi} a y_{k} e^{\frac{\left(a y_{k}\right)^{2}}{2}}}{1+\left[P_{f_{k}}-Q\left(a y_{k}\right)\right] \sqrt{2 \pi} a y_{k} e^{\frac{\left(a y_{k}\right)^{2}}{2}}}\right\} .
\end{aligned}
$$

Proof: Under hypothesis $H_{1}$, we have

$$
\begin{aligned}
f\left(y_{k} \mid H_{1}\right)= & \frac{\sum_{u_{k}}\left[p\left(H_{1}\right) p\left(u_{k} \mid H_{1}\right) f\left(y_{k} \mid u_{k}\right)\right]}{p\left(H_{1}\right)} \\
= & \sum_{u_{k}}\left[p\left(u_{k} \mid H_{1}\right) f\left(y_{k} \mid u_{k}\right)\right] \\
= & P_{d_{k}} f\left(y_{k} \mid u_{k}=1\right)+\left(1-P_{d_{k}}\right) f\left(y_{k} \mid u_{k}=-1\right) \\
= & \frac{2 \sigma}{\sqrt{2 \pi}\left(1+2 \sigma^{2}\right)} e^{-\frac{y_{k}^{2}}{2 \sigma^{2}}} \\
& \times\left\{1+\left[P_{d_{k}}-Q\left(a y_{k}\right)\right] \sqrt{2 \pi} a y_{k} e^{\frac{\left(a y_{k}\right)^{2}}{2}}\right\}
\end{aligned}
$$

where (1), Lemma 1, and the identity $Q\left(a y_{k}\right)=1-Q\left(-a y_{k}\right)$ have been used.
Following the same procedure and replacing $P_{d_{k}}$ with $P_{f_{k}}$, we obtain $f\left(y_{k} \mid H_{0}\right)$ as follows:

$$
\begin{aligned}
f\left(y_{k} \mid H_{0}\right)= & \frac{2 \sigma}{\sqrt{2 \pi}\left(1+2 \sigma^{2}\right)} e^{-\frac{y_{k}^{2}}{2 \sigma^{2}}} \\
& \times\left\{1+\left[P_{f_{k}}-Q\left(a y_{k}\right)\right] \sqrt{2 \pi} a y_{k} e^{\frac{\left(a y_{k}\right)^{2}}{2}}\right\} .
\end{aligned}
$$

Obviously, the likelihood ratio is

$$
\begin{aligned}
\frac{f\left(\mathbf{y} \mid H_{1}\right)}{f\left(\mathbf{y} \mid H_{0}\right)} & =\prod_{k=1}^{K} \frac{f\left(y_{k} \mid H_{1}\right)}{f\left(y_{k} \mid H_{0}\right)} \\
& =\prod_{k=1}^{K} \frac{1+\left[P_{d_{k}}-Q\left(a y_{k}\right)\right] \sqrt{2 \pi} a y_{k} e^{\frac{\left(a y_{k}\right)^{2}}{2}}}{1+\left[P_{f_{k}}-Q\left(a y_{k}\right)\right] \sqrt{2 \pi} a y_{k} e^{\frac{\left(a y_{k}\right)^{2}}{2}}}
\end{aligned}
$$

where assumptions of the conditional independence of local decisions and the independence of different fading channels have been used. Taking logarithm of the above equation on both sides, we obtain the log-likelihood ratio $\Lambda_{4}$ given in (12). $\quad$ Q.E.D.

As we can see, this fusion statistic requires the knowledge of channel statistics and local sensor detection performance indices. To distinguish this new fusion rule from the optimal LR-based fusion rule that requires instantaneous CSI, we call it the likelihood ratio test based on channel statistics (LRT-CS).

Next, we determine the high- and low-SNR approximations of the LRT-CS fusion statistic. These approximations are derived and stated in the following two propositions.

Proposition 1: As the channel noise variance $\sigma^{2} \rightarrow 0$, i.e., $\mathrm{SNR} \rightarrow \infty, \Lambda_{4}$ defined in (12) reduces to $\Lambda_{1}$ defined in (4), i.e.,

$$
\lim _{\sigma^{2} \rightarrow 0} \Lambda_{4}=\Lambda_{1} .
$$

Proof: As $\sigma^{2} \rightarrow 0, a=1 /\left(\sigma \sqrt{1+2 \sigma^{2}}\right) \rightarrow \infty$. Define $\mathcal{S}_{0}=\left\{k: y_{k}<0\right\}$ and $\mathcal{S}_{1}=\left\{k: y_{k}>0\right\}$, and we rewrite $\Lambda_{4}$ as

$$
\begin{aligned}
\Lambda_{4}=\sum_{k \in \mathcal{S}_{0}} \log \left\{\frac{1+\left[P_{d_{k}}-Q\left(a y_{k}\right)\right] \sqrt{2 \pi} a y_{k} e^{\frac{\left(a y_{k}\right)^{2}}{2}}}{1+\left[P_{f_{k}}-Q\left(a y_{k}\right)\right] \sqrt{2 \pi} a y_{k} e^{\frac{\left(a y_{k}\right)^{2}}{2}}}\right\} \\
+\sum_{k \in \mathcal{S}_{1}} \log \left\{\frac{1+\left[P_{d_{k}}-Q\left(a y_{k}\right)\right] \sqrt{2 \pi} a y_{k} e^{\frac{\left(a y_{k}\right)^{2}}{2}}}{1+\left[P_{f_{k}}-Q\left(a y_{k}\right)\right] \sqrt{2 \pi} a y_{k} e^{\frac{\left(a y_{k}\right)^{2}}{2}}}\right\} .
\end{aligned}
$$

Given that $a \rightarrow \infty$ (high SNR assumption), we have, for $k \in$ $\mathcal{S}_{0}, Q\left(a y_{k}\right) \rightarrow Q(-\infty)=1$, and $\left|a y_{k} e^{\left(a y_{k}\right)^{2} / 2}\right| \gg 1$; while for $k \in \mathcal{S}_{1}, Q\left(a y_{k}\right) \rightarrow Q(\infty)=0$, and $a y_{k} e^{\left(a y_{k}\right)^{2} / 2} \gg 1$. Hence, the assumption $\sigma^{2} \rightarrow 0$ can be used to obtain

$$
\lim _{\sigma^{2} \rightarrow 0} \Lambda_{4}=\sum_{k \in \mathcal{S}_{0}} \log \left[\frac{1-P_{d_{k}}}{1-P_{f_{k}}}\right]+\sum_{k \in \mathcal{S}_{1}} \log \left[\frac{P_{d_{k}}}{P_{f_{k}}}\right]=\Lambda_{1}
$$

which is exactly the Chair-Varshney decision fusion statistic. Q.E.D.

Proposition 2: As $\sigma^{2} \rightarrow \infty, \Lambda_{4}$ in (12) reduces to

$$
\hat{\Lambda}_{3}=\sum_{k=1}^{K}\left(P_{d_{k}}-P_{f_{k}}\right) y_{k} \text {. }
$$


Further, if the local sensors are identical, i.e., $P_{d_{k}}=P_{d}$ and $P_{f_{k}}=P_{f}$ for all $k$, and $P_{d}>P_{f}$, then $\Lambda_{4}$ further reduces to a form analogous to an EGC statistic

$$
\Lambda_{3}=\frac{1}{K} \sum_{k=1}^{K} y_{k} .
$$

Proof: For low SNR, i.e., $\sigma^{2} \rightarrow \infty, a=1 /$ $\left(\sigma \sqrt{1+2 \sigma^{2}}\right) \rightarrow 0$. The Taylor series expansion of $Q(x)$ is

$$
Q(x)=\frac{1}{2}-\frac{1}{\sqrt{2 \pi}}\left(x-\frac{x^{3}}{2}+\frac{x^{5}}{2 \cdot 4}-\frac{x^{7}}{2 \cdot 4 \cdot 8}+\cdots\right) .
$$

Therefore, $\lim _{a \rightarrow 0} Q\left(a y_{k}\right) \approx 1 / 2-a y_{k} / \sqrt{2 \pi}$. In addition, $e^{\left(a y_{k}\right)^{2} / 2}$ can be approximated by the first-order Taylor series expansion, i.e., $e^{\left(a y_{k}\right)^{2} / 2} \approx 1+a^{2} y_{k}^{2} / 2$. Hence

$$
\begin{aligned}
\lim _{a \rightarrow 0}\{1+ & {\left.\left[P_{d_{k}}-Q\left(a y_{k}\right)\right] \sqrt{2 \pi} a y_{k} e^{\frac{\left(a y_{k}\right)^{2}}{2}}\right\} } \\
=\lim _{a \rightarrow 0} & \left\{1+\left[P_{d_{k}}-\left(\frac{1}{2}-\frac{a y_{k}}{\sqrt{2 \pi}}\right)\right] \sqrt{2 \pi} a y_{k}\right. \\
& \left.\times\left(1+\frac{a^{2} y_{k}^{2}}{2}\right)\right\} \\
= & \lim _{a \rightarrow 0}\left\{1+\left(P_{d_{k}}-\frac{1}{2}\right) \sqrt{2 \pi} a y_{k}\right\}
\end{aligned}
$$

where higher order terms have been ignored.

Similarly

$$
\begin{aligned}
& \lim _{a \rightarrow 0}\left\{1+\left[P_{f_{k}}-Q\left(a y_{k}\right)\right] \sqrt{2 \pi} a y_{k} e^{\frac{\left(a y_{k}\right)^{2}}{2}}\right\} \\
& =\lim _{a \rightarrow 0}\left\{1+\left(P_{f_{k}}-\frac{1}{2}\right) \sqrt{2 \pi} a y_{k}\right\} .
\end{aligned}
$$

With the above results, it is straightforward to see that

$$
\begin{aligned}
\lim _{a \rightarrow 0} \Lambda_{4}= & \lim _{a \rightarrow 0} \sum_{k=1}^{K} \log \left\{\frac{1+\left(P_{d_{k}}-\frac{1}{2}\right) \sqrt{2 \pi} a y_{k}}{1+\left(P_{f_{k}}-\frac{1}{2}\right) \sqrt{2 \pi} a y_{k}}\right\} \\
= & \lim _{a \rightarrow 0} \sum_{k=1}^{K}\left[\left(P_{d_{k}}-\frac{1}{2}\right) \sqrt{2 \pi} a y_{k}\right] \\
& -\lim _{a \rightarrow 0} \sum_{k=1}^{K}\left[\left(P_{f_{k}}-\frac{1}{2}\right) \sqrt{2 \pi} a y_{k}\right] \\
= & \lim _{a \rightarrow 0} \sum_{k=1}^{K}\left(P_{d_{k}}-P_{f_{k}}\right) \sqrt{2 \pi} a y_{k}
\end{aligned}
$$

where we have used the fact $\lim _{x \rightarrow 0} \log (1+x)=\lim _{x \rightarrow 0} x$. This statistic is equivalent to $\hat{\Lambda}_{3}$. Further, if $P_{d_{k}}=P_{d}$ and $P_{f_{k}}=P_{f}$ for all $k$, the fusion statistic can be further reduced to $\Lambda_{3}$ as in (7).

Q.E.D.

Thus, under the identical local sensor assumption, the EGC statistic is a low-SNR approximation to the LRT-CS decision fusion statistic. Therefore, we have a theoretical justification of the EGC statistic which was proposed as a heuristic alternative to the MRC statistic in [17].

\section{Performance AnAlysis}

\section{A. The Distribution of the Chair-Varshney Statistic}

In the development of the LRT-CS fusion rule, we have already obtained the pdf of $f\left(y_{k} \mid H_{1}\right)$ and $f\left(y_{k} \mid H_{0}\right)$. With these pdfs available, it is possible to derive the closed-form distribution of the Chair-Varshney statistic, which is very helpful for performance analysis. Define $K_{0}=\left|\mathcal{S}_{0}\right|$, where $\mathcal{S}_{0}=\{k$ : $\left.y_{k}<0\right\}$, i.e., $K_{0}$ is the cardinality of $\mathcal{S}_{0}$, and $K_{1}=\left|\mathcal{S}_{1}\right|$, where $\mathcal{S}_{1}=\left\{k: y_{k} \geq 0\right\}$. Thus, $K_{1}+K_{0}=K$. With these definitions and assuming that all the sensors are identical, meaning $P_{d_{k}}=P_{d}$ and $P_{f_{k}}=P_{f}$ for all $k$, (4) becomes

$$
\begin{aligned}
\Lambda_{1} & =\log \left(\frac{P_{d}}{P_{f}}\right) K_{1}+\log \left(\frac{1-P_{d}}{1-P_{f}}\right)\left(K-K_{1}\right) \\
& =\log \left[\frac{P_{d}\left(1-P_{f}\right)}{P_{f}\left(1-P_{d}\right)}\right] K_{1}+\log \left(\frac{1-P_{d}}{1-P_{f}}\right) K
\end{aligned}
$$

which is an affine function of $K_{1}$. When $\left(P_{d}\left(1-P_{f}\right)\right) /$ $\left(P_{f}\left(1-P_{d}\right)\right)>1$ (or $P_{d}>P_{f}$ ), the statistic $\Lambda_{1}$ is equivalent to $K_{1}$. When all the sensors are identical, all $y_{k}$ 's are independent and identically distributed (i.i.d.). Therefore, $K_{1}$ is a binomial $(K, p)$ distribution, where the success probability $p$ is defined as

$$
p=P\left(y_{k} \geq 0\right) .
$$

We denote $p_{1}$ and $p_{0}$ as the success probabilities under $H_{1}$ and $H_{0}$, respectively. The closed-form solutions are provided in the following Lemma.

Lemma 2: The probabilities of a nonnegative observation $y_{k}$ under hypotheses $H_{1}$ and $H_{0}$ are, respectively

$$
p_{1}=p\left(y_{k} \geq 0 \mid H_{1}\right)=\frac{1}{2}+\frac{P_{d}-\frac{1}{2}}{\sqrt{1+2 \sigma^{2}}}
$$

and

$$
p_{0}=p\left(y_{k} \geq 0 \mid H_{0}\right)=\frac{1}{2}+\frac{P_{f}-\frac{1}{2}}{\sqrt{1+2 \sigma^{2}}} .
$$

Proof: See Appendix II.

System-level detection performance can be evaluated exactly by using the Binomial distribution, namely

$$
P_{d_{0}}=\sum_{i=K_{\tau}}^{K}\left(\begin{array}{c}
K \\
i
\end{array}\right) p_{1}^{i}\left(1-p_{1}\right)^{K-i}
$$

where $K_{\tau}$ is the threshold. For the same $K_{\tau}$, the corresponding $P_{f_{0}}$ is

$$
P_{f_{0}}=\sum_{i=K_{\tau}}^{K}\left(\begin{array}{c}
K \\
i
\end{array}\right) p_{0}^{i}\left(1-p_{0}\right)^{K-i} .
$$

As we can see, there are a total of $K+2$ pairs of $P_{d_{0}}$ and $P_{f_{0}}$ as $K_{\tau}$ takes values from 0 to $K+1$. The receiver operating characteristic (ROC) curve obtained by (23) and (24) is indistinguishable from that obtained by Monte Carlo simulations. In this paper, we do not give the figure containing these ROC curves due to limited space. 


\section{B. Performance Analysis Using Deflection Coefficient}

If we assume that all the sensors are identical (thus $P_{f_{k}}=P_{f}$ and $P_{d_{k}}=P_{d}$ for all $k$ ), all the decision statistics we have discussed in Sections II.B and III are sums of i.i.d. random variables which allows a direct application of the central limit theorem (CLT). Therefore, if the number of sensors is large, all these statistics can be approximated by Gaussian distributions. This makes the comparisons and analysis much easier.

However, the accuracy of the Gaussian approximation is directly related to the number of sensors employed. When the number of sensors is small, there is always a discrepancy between the ROC curves obtained by Monte Carlo simulation and those by analytical approximation using the CLT. In addition, we have found through extensive simulations that the accuracy of the CLT approximation not only depends on the number of sensors involved, but also on other system parameters, such as the sensor performance indexes $\left(P_{d_{k}}\right.$ and $\left.P_{f_{k}}\right)$ and the channel SNR. In addition to using the ROC curve for performance comparison, one can also resort to the so-called deflection coefficient [20], [21], especially when the statistical properties of the signal and noise are limited to moments up to a given order. The deflection coefficient is defined as

$$
D(\Lambda)=\frac{\left[E\left(\Lambda \mid H_{1}\right)-E\left(\Lambda \mid H_{0}\right)\right]^{2}}{\operatorname{Var}\left(\Lambda \mid H_{0}\right)} .
$$

In the case of $\operatorname{Var}\left(\Lambda \mid H_{1}\right)=\operatorname{Var}\left(\Lambda \mid H_{0}\right)$, this is in essence the SNR of the detection statistic. It is worth noting that the use of deflection criterion leads to the optimum LR receiver in many cases of practical importance [20]. For example, in the problem of detecting a Gaussian signal in Gaussian noise, an LR detector is obtained by maximizing the deflection measure.

In order to calculate the deflection coefficient, we need the first and second order statistics of the fusion statistic (mean and variance). In the case of the LRT-CS fusion statistic, the mean is found in (26), shown at the bottom of the page, where

$$
g(y)=\log \left\{\frac{1+\left[P_{d}-Q(a y)\right] \sqrt{2 \pi} a y e^{\frac{(a y)^{2}}{2}}}{1+\left[P_{f}-Q(a y)\right] \sqrt{2 \pi} a y e^{\frac{(a y)^{2}}{2}}}\right\} .
$$

Note that whenever it does not cause confusion, the subscript $k$ is dropped for simplicity. Similarly

$$
\begin{aligned}
\operatorname{Var}\left[\frac{\Lambda_{4}}{K} \mid H_{1}\right] & =\frac{1}{K} \operatorname{Var}\left[g(y) \mid H_{1}\right] \\
& =\frac{1}{K}\left\{E\left[g^{2}(y) \mid H_{1}\right]-\mu_{\mathrm{LRC}_{1}}^{2}\right\} \\
& \triangleq \sigma_{\mathrm{LRC}_{1}}^{2}
\end{aligned}
$$

where

$$
\begin{aligned}
E\left[g^{2}(y) \mid H_{1}\right] & =\int_{-\infty}^{\infty} g^{2}(y) \frac{2 \sigma}{\sqrt{2 \pi}\left(1+2 \sigma^{2}\right)} e^{-\frac{y^{2}}{2 \sigma^{2}}} \\
& \times\left\{1+\left[P_{d}-Q(a y)\right] \sqrt{2 \pi} a y e^{\frac{(a y)^{2}}{2}}\right\} d y .
\end{aligned}
$$

While it is nearly impossible to obtain closed-form solutions of (26) and (29), numerical integration can be employed for the computation of these expressions. The mean and variance under $H_{0}$ can be calculated following a similar procedure.

With regard to the Chair-Varshney statistic, we have shown in Section IV.A that $\Lambda_{2}$ is equivalent to $K_{1}$, which is binomial distributed. Therefore, $K_{1}$ can be viewed as the sum of $K$ i.i.d. Bernoulli random variables with success probabilities $p_{1}$ and $p_{0}$, under hypothesis $H_{1}$ and $H_{0}$, respectively. Given $p_{1}$ and $p_{0}$, it is easy to show that

$$
\begin{aligned}
\mu_{\mathrm{CV}_{1}} & =E\left[K_{1} \mid H_{1}\right] \\
& =K\left(\frac{1}{2}+\frac{P_{d}-\frac{1}{2}}{\sqrt{1+2 \sigma^{2}}}\right)
\end{aligned}
$$

and

$$
\begin{aligned}
\sigma_{\mathrm{CV}_{1}}^{2} & =\operatorname{Var}\left[K_{1} \mid H_{1}\right] \\
& =K\left[\frac{1}{4}-\frac{\left(P_{d}-\frac{1}{2}\right)^{2}}{1+2 \sigma^{2}}\right] .
\end{aligned}
$$

Similarly

and

$$
\mu_{\mathrm{CV}_{0}}=K\left(\frac{1}{2}+\frac{P_{f}-\frac{1}{2}}{\sqrt{1+2 \sigma^{2}}}\right)
$$

$$
\sigma_{\mathrm{CV}_{0}}^{2}=K\left[\frac{1}{4}-\frac{\left(P_{f}-\frac{1}{2}\right)^{2}}{1+2 \sigma^{2}}\right] \text {. }
$$

$$
\begin{aligned}
E\left[\frac{\Lambda_{4}}{K} \mid H_{1}\right]= & \frac{1}{K} \sum_{k=1}^{K} E\left[\log \left\{\frac{1+\left[P_{d_{k}}-Q\left(a y_{k}\right)\right] \sqrt{2 \pi} a y_{k} e^{\frac{\left(a y_{k}\right)^{2}}{2}}}{1+\left[P_{f_{k}}-Q\left(a y_{k}\right)\right] \sqrt{2 \pi} a y_{k} e^{\frac{\left(a y_{k}\right)^{2}}{2}}}\right\} \mid H_{1}\right] \\
= & E\left[g(y) \mid H_{1}\right] \\
= & \int_{-\infty}^{\infty} g(y) \frac{2 \sigma}{\sqrt{2 \pi}\left(1+2 \sigma^{2}\right)} e^{-\frac{y^{2}}{2 \sigma^{2}}} \\
& \times\left\{1+\left[P_{d_{k}}-Q(a y)\right] \sqrt{2 \pi} a y e^{\frac{(a y)^{2}}{2}}\right\} d y \\
\triangleq & \mu_{\mathrm{LRC}_{1}} .
\end{aligned}
$$


TABLE I

COMPARISON AMONG FIVE DIFFERENT Fusion RULES

\begin{tabular}{|c||c|c|}
\hline Fusion statistic & a priori information required & Performance \\
\hline$\Lambda$ (LR) & Channel SNR and sensor performance indices & Optimum \\
\hline$\Lambda_{1}$ (Chair-Varshney) & Sensor performance indices & Near-optimal for large SNR \\
\hline$\Lambda_{2}$ (MRC) & Channel SNR & Near-optimal for low SNR \\
\hline$\Lambda_{3}$ (EGC) & None & Robust for most SNR range \\
\hline$\Lambda_{4}$ (LRT-CS) & Channel statistics and sensor performance indices & Near-optimal \\
\hline
\end{tabular}

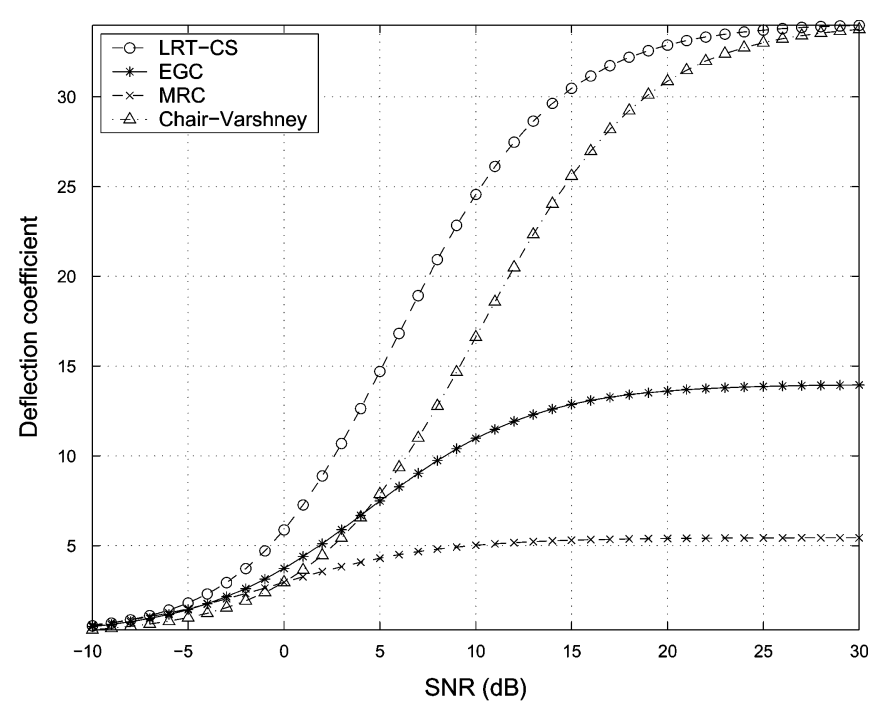

Fig. 2. Deflection coefficient for different statistics. There are eight sensors with $P_{d}=0.5$ and $P_{f}=0.05$.

The closed-form mean and variance for EGC and MRC statistics are available in [17]. Given these statistics, we calculate the deflection coefficient at different channel SNR for different fusion statistics. This helps us understand how the detection performance varies for different values of channel SNR. As we can see from Fig. 2, at very low channel SNR, the performance of the LRT-CS statistic reduces to the EGC statistic; for high-channel SNR, the Chair-Varshney statistic has the same performance as the LRT-CS statistic. The LRT-CS fusion rule is optimal when only channel statistics and sensor performance indexes are available. The LRT-CS fusion statistic has a larger deflection coefficient than other suboptimal fusion statistics. The only exception is when SNR is very low (less than $-8 \mathrm{~dB}$ ). In this case, the MRC statistic outperforms the LRT-CS statistic. Again, this is because the MRC statistic uses more prior information, namely the instantaneous CSI.

\section{Simulation Results}

In the last section, we have compared the performance of different fusion rules in terms of the deflection coefficient. Even though it is an interesting metric and it completely characterizes the detection performance under the Gaussian assumption, in general, it can not be proved that a greater deflection always leads to a better performance in terms of ROC curves. Therefore, in this section, simulations are employed to obtain

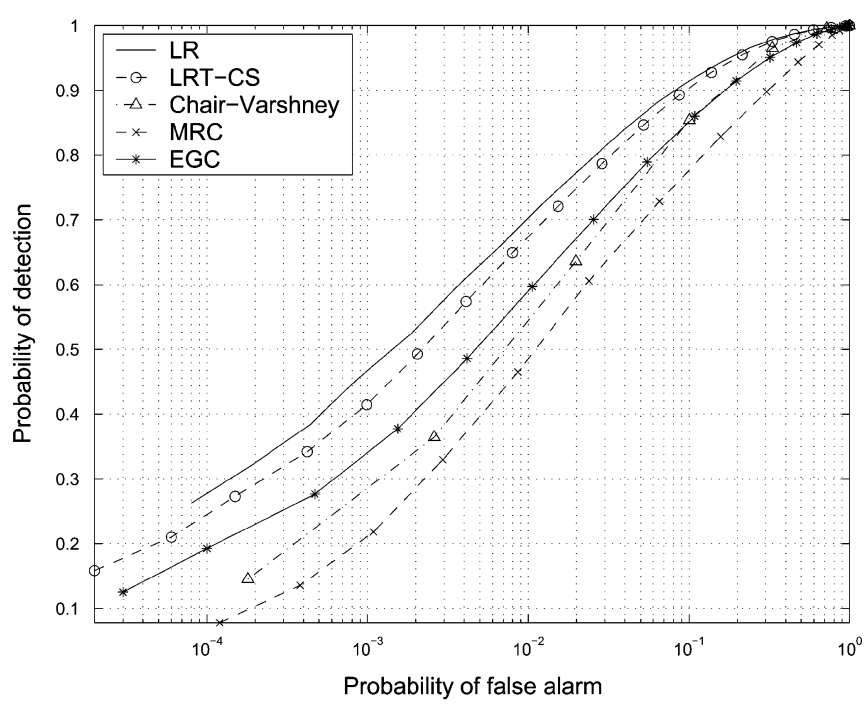

Fig. 3. ROC curves for various fusion statistics for the Rayleigh fading channel with average channel SNR $=5 \mathrm{~dB}$. There are eight sensors with $P_{d}=0.5$ and $P_{f}=0.05$.

the ROC curves for different fusion rules. Note that each fusion rule requires different amount of prior information about the sensor performance and the fading channels, as summarized in the second column of Table I.

Fig. 3 gives the ROC curves corresponding to different fusion statistics at channel SNR of $5 \mathrm{~dB}$. Each sensor's false alarm rate is assumed to be $P_{f}=0.05$ while the detection probability is $P_{d}=0.5$. The total number of sensors is fixed at eight.

The optimal LR-based fusion rule provides the uniformly most powerful detection performance, however it requires instantaneous CSI. On the other hand, its performance can be approached closely by the LRT-CS fusion rule. The performance of the LRT-CS fusion rule is slightly worse than the optimal LR-based fusion rule with instantaneous CSI and is better than the three suboptimal schemes.

To better understand the performance difference as a function of channel SNR, Fig. 4 gives the probability of detection as a function of the average channel SNR for a constant system false alarm rate of $P_{f_{0}}=0.01$. The parameter setting is identical to the above example. From this figure, it is clear that performances of EGC and the Chair-Varshney fusion rules approach that of the LRT-CS fusion rule at very low and very high SNR respectively, which is consistent with the results of Fig. 2 using the deflection coefficient for the same set of parameters. The jumpy behavior of the Chair-Varshney approach can be explained easily. As we have discussed in Section IV-A, $K_{1}$, 


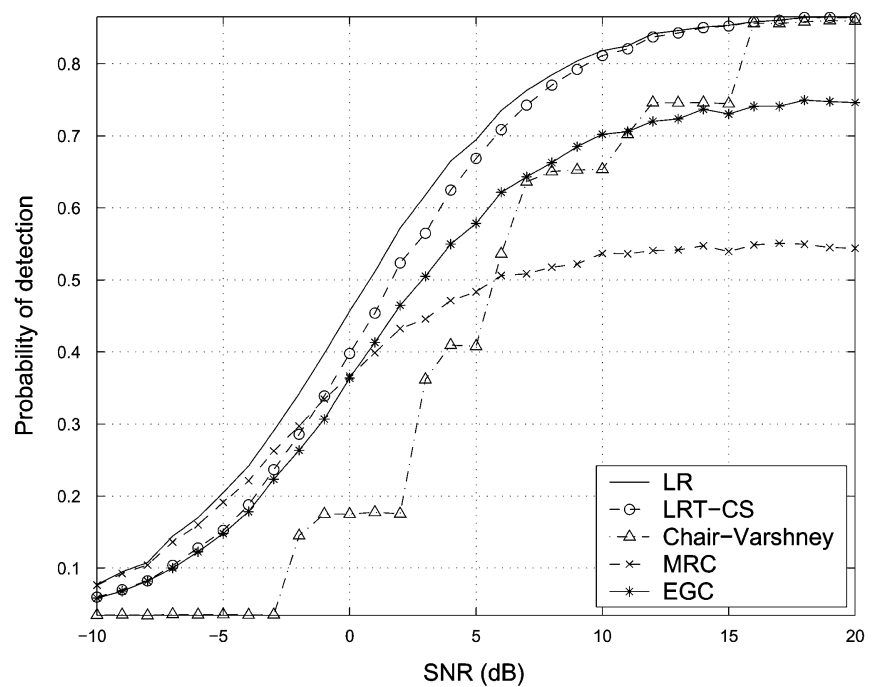

Fig. 4. System-level probability of detection as a function of average channel SNR for Rayleigh fading channels with eight sensors whose $P_{d}=0.5$ and $P_{f}=0.05$. The system false alarm rate is fixed at $P_{f_{0}}=0.01$.

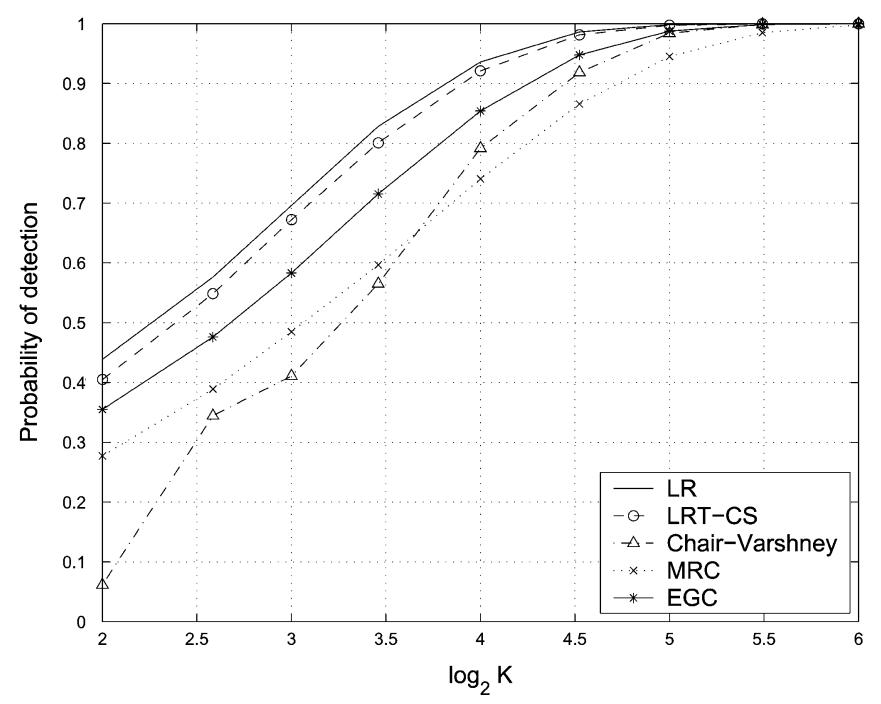

Fig. 5. System-level probability of detection as a function of number of sensors $K . P_{d}=0.5, P_{f}=0.05$, and the average channel SNR is $5 \mathrm{~dB}$. The system false alarm rate is fixed at $P_{f_{0}}=0.01$.

which is equivalent to the Chair-Varshney statistic, is binomial distributed. With a local performance index $P_{d}$ set as 0.5 , from (21), it is clear that the success probability of the Binomial distribution under hypothesis $H_{1}\left(p_{1}\right)$ is a constant $(0.5)$ and independent of channel SNR. Therefore, in this special case, according to (23), the system level $P_{d_{0}}$ is only a function of the threshold $K_{\tau}$. As we know, there are only finite integer numbers (from 0 to $K+1$ ) from which $K_{\tau}$ can take values. The value of $K_{\tau}$ is determined by the average channel SNR and the system level false alarm rate $P_{f_{0}}$ (see (22) and (24)). Even for different SNR values, and hence different $p_{0}$ values, $K_{\tau}$ could still be the same due to its finite alphabet property. Thus, with the same $K_{\tau}, P_{d_{0}}$ remains constant for a certain range of channel SNRs.

The system performance (probability of detection) as a function of total number of sensors $(K)$ is shown in Fig. 5. As we can see, when $K$ is very large, the probability of detection at the fusion center approaches 1 , even when the local sensors have a modest detection performance with $P_{d}=0.5$ and $P_{f}=0.05$, and the average channel SNR is very low $(5 \mathrm{~dB})$. This is due to the accumulation of information from a large number of sensors. Note that no matter how many sensors are employed, the LRT-CS fusion rule outperforms the other three sub-optimum fusion rules. With the specific system parameters in this example, the performance of the EGC fusion rule is quite robust and outperforms both MRC and Chair-Varshney fusion rules, regardless of the scaling factor $K$.

The tradeoff between detection performance and the requirement on a priori information for each of the fusion schemes is summarized in Table I. The summary is also based on the assumption that all sensors' performance indexes are identical to each other.

\section{Sensors With Different Detection Performances and Channel SNRS}

In Sections IV-A, IV-B, and IV-C, we gave examples where the channel SNR and the detection performance are assumed identical for all sensors. In practice, however, it is often the case that different sensors have different channel SNRs and different detection performances. In this section, we investigate and compare the performances of different fusion rules in these more complicated and practical scenarios.

First, we assume that all the wireless channels between local sensors and the fusion center have the same average SNR. There are totally $K=8$ sensors. All the sensors have the same probability of false alarm, and $P_{f}=0.05$. However, they have different probabilities of detection. In this particular example, we assume that $\vec{P}_{d}=[0.1,0.2, \ldots, 0.8]$, where $\overrightarrow{P_{d}}=\left[P_{d_{1}}, P_{d_{2}}, \ldots, P_{d_{K}}\right]$. The simulation results are shown in Fig. 6 .

Next, we assume that all the sensors have the same detection performance indexes $\left(P_{d}=0.5\right.$ and $\left.P_{f}=0.05\right)$. However, different sensors have different average channel SNRs for their channels to the fusion center. We assume that there are $K=8$ sensors, and $\vec{S}=[\bar{S}-6, \bar{S}-4, \bar{S}-2, \bar{S}, \bar{S}, \bar{S}+2, \bar{S}+4, \bar{S}+6]$ $\mathrm{dB}$, where $\vec{S}=\left[\mathrm{SNR}_{1}, \mathrm{SNR}_{2}, \ldots, \mathrm{SNR}_{K}\right] \mathrm{dB}$, and $\bar{S}$ is the arithmetic mean of all the average channel SNRs (in decibels). For example, if $\bar{S}=10 \mathrm{~dB}$, then $\vec{S}=[4,6,8,10,10,12,14,16]$ $\mathrm{dB}$. Note that there are significant variations for the average channel SNRs. There is 12-dB difference between the largest and the smallest average channel SNRs. In Fig. 7, the systemlevel probability of detection is plotted as a function of the mean value of the average SNRs of all the channels $(\bar{S})$.

From both Figs. 6 and 7, we can see that the LRT-CS fusion rule has much better performance than EGC and Chair-Varshney rules. It outperforms MRC except when the channel SNR is very low (see Fig. 6). With low-channel SNR (or average channel SNR) values, the MRC fusion rule has the best performance among the three suboptimal fusion rules. With high-channel SNR (or average channel SNR) values, the Chair-Varshney fusion rule has the best performance among the three suboptimal fusion rules. The EGC has a very robust performance when channel SNR is low or medium. 


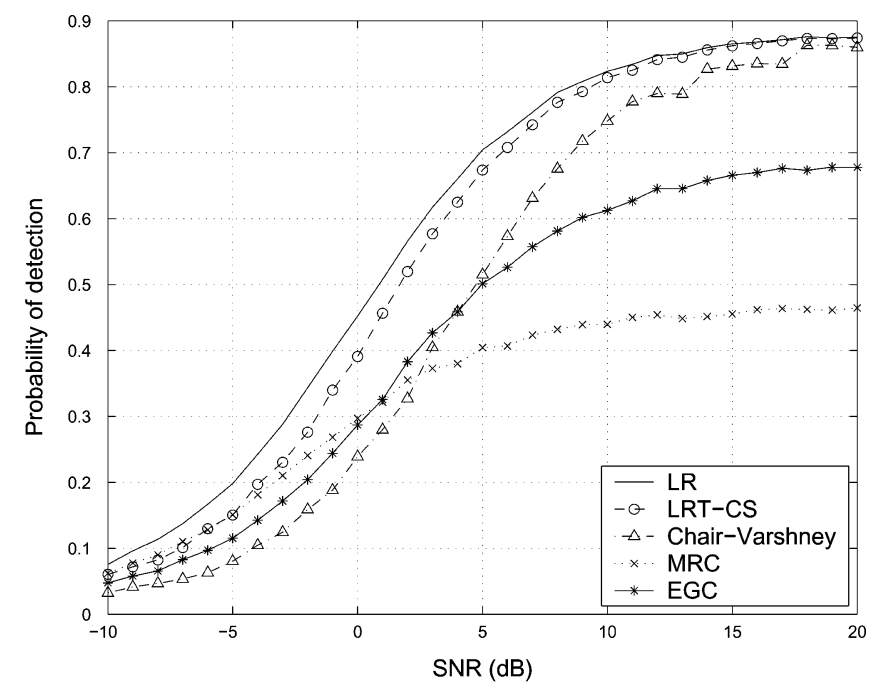

Fig. 6. System-level probability of detection as a function of average channel SNR for Rayleigh fading channels with eight sensors whose detection performances are different. The system false alarm rate is fixed at $P_{f_{0}}=0.01$.

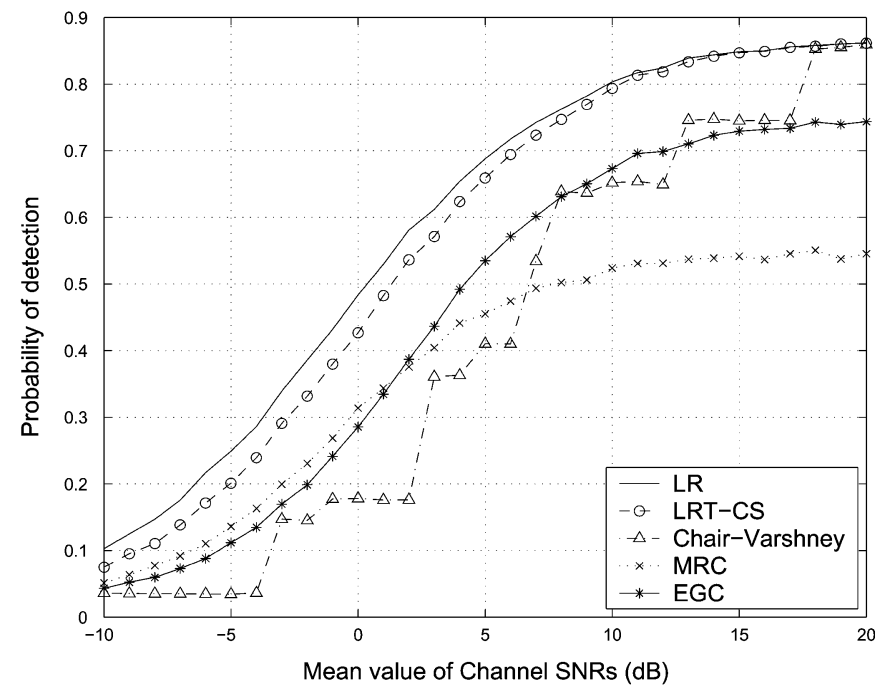

Fig. 7. System-level probability of detection versus the mean value of average SNRs of the Rayleigh fading channels with eight sensors, whose average channel SNRs are different. $P_{d}=0.5$ and $P_{f}=0.05$. The system false alarm rate is fixed at $P_{f_{0}}=0.01$.

\section{SUMMARY}

The problem of fusing binary decisions transmitted over fading and noisy channels in a WSN was revisited in this paper. Along with various fusion rules obtained previously, we presented a new LR-based test (LRT-CS) which requires only the knowledge of the statistical characteristics of the wireless channel instead of instantaneous CSI. The Chair-Varshney fusion statistic provides high SNR approximation to the new fusion statistic, while the statistic in the form of an EGC gives a low SNR approximation. The new fusion rule outperforms both the EGC and Chair-Varshney fusion rules, has better performance than the MRC fusion rule for most practical SNR values (except for very low SNR values), and degrades performance slightly compared with the optimal LR-based fusion rule that requires instantaneous CSI. In addition, we derived a closed-form solution to the distribution of the Chair-Varshney statistic, which facilitates the performance analysis and comparison.

Analysis based on deflection coefficient was carried out to intuitively illustrate the performance advantage of the new fusion rule over the other suboptimal rules. Simulation results were given for performance comparison and were consistent with the analysis. Cases where different sensors have different detection performances or different channel SNRs were also investigated to illustrate the robustness of the proposed LRT-CS fusion rule.

\section{APPENDIX A \\ PROOF OF LEMMA 1}

Substituting (9) into (11), setting $u_{k}=1$ and considering that $n_{k}$ is a Gaussian random variable with zero mean and variance $\sigma^{2}$, we have

$$
\begin{aligned}
& f\left(y_{k} \mid u_{k}=1\right)=\int_{0}^{\infty} 2 x e^{-x^{2}} \frac{1}{\sqrt{2 \pi} \sigma} e^{-\frac{(x-y)^{2}}{2 \sigma^{2}}} d x \\
& =\frac{2}{\sqrt{2 \pi} \sigma} e^{-\frac{y^{2}}{1+2 \sigma^{2}}} \int_{0}^{\infty} x \exp \left[-\frac{\left(x-\frac{y}{1+2 \sigma^{2}}\right)^{2}}{2 \frac{\sigma^{2}}{1+2 \sigma^{2}}}\right] d x .
\end{aligned}
$$

By plugging $t=x-y /\left(1+2 \sigma^{2}\right)$ into the above equation, we obtain

$$
\begin{aligned}
f\left(y_{k} \mid u_{k}=1\right) \\
=\frac{2}{\sqrt{2 \pi} \sigma} e^{-\frac{y^{2}}{1+2 \sigma^{2}}} \\
\quad \times \int_{-\frac{y}{1+2 \sigma^{2}}}^{\infty}\left(t+\frac{y}{1+2 \sigma^{2}}\right) \exp \left[-\frac{t^{2}}{2 \frac{\sigma^{2}}{1+2 \sigma^{2}}}\right] d t \\
=\frac{2 \sigma}{\sqrt{2 \pi}\left(1+2 \sigma^{2}\right)} e^{-\frac{y^{2}}{2 \sigma^{2}}}\left[1+\sqrt{2 \pi} a y e^{\frac{(a y)^{2}}{2}} Q(-a y)\right]
\end{aligned}
$$

where $a=1 /\left(\sigma \sqrt{\left(1+2 \sigma^{2}\right)}\right)$.

Similarly, for the case of $u_{k}=-1$, we have

$$
\begin{aligned}
f\left(y_{k} \mid u_{k}=-1\right)= & \frac{2 \sigma}{\sqrt{2 \pi}\left(1+2 \sigma^{2}\right)} \\
& \times e^{-\frac{y^{2}}{2 \sigma^{2}}}\left[1-\sqrt{2 \pi} a y e^{\frac{(a y)^{2}}{2}} Q(a y)\right] .
\end{aligned}
$$

\section{APPENDIX B}

\section{PROOF OF LEMMA 2}

$$
\begin{aligned}
p_{1}= & p\left(y_{k} \geq 0 \mid H_{1}\right) \\
= & \int_{0}^{\infty} \frac{2 \sigma}{\sqrt{2 \pi}\left(1+2 \sigma^{2}\right)} \\
& \times e^{-\frac{y^{2}}{2 \sigma^{2}}\left\{1+\left[P_{d}-Q(a y)\right] \sqrt{2 \pi} a y e^{\frac{(a y)^{2}}{2}}\right\} d y} \\
= & \frac{2 \sigma \quad}{\sqrt{2 \pi}\left(1+2 \sigma^{2}\right)}\left[\frac{\sqrt{2 \pi} \sigma}{2}+\frac{\sqrt{2 \pi} P_{d} \sqrt{1+2 \sigma^{2}}}{2 \sigma}\right. \\
& \left.\quad+\frac{\sqrt{2 \pi}\left(1-\sqrt{1+2 \sigma^{2}}\right)}{4 \sigma}\right] \\
= & \frac{1}{2}+\frac{P_{d}-\frac{1}{2}}{\sqrt{1+2 \sigma^{2}}} .
\end{aligned}
$$


Following a similar procedure and replacing $P_{d}$ with $P_{f}$, it is easy to obtain

$$
p_{0}=\frac{1}{2}+\frac{P_{f}-\frac{1}{2}}{\sqrt{1+2 \sigma^{2}}} .
$$

\section{REFERENCES}

[1] C. Intanagonwiwat, R. Govindan, D. Estrin, J. Heidemann, and F. Silva, "Directed diffusion for wireless sensor networking," IEEE/ACM Trans. Netw., vol. 11, no. I, pp. 2-16, Feb. 2003.

[2] IEEE Signal Processing Mag. (Special Issue on Collaborative Signal and Information Processing in Microsensor Networks), vol. 19, no. 2, Mar. 2002.

[3] Z. Chair and P. K. Varshney, "Optimal data fusion in multiple sensor detection systems," IEEE Trans. Aerosp. Electron. Syst., vol. 22, no. 1, pp. 98-101, Jan. 1986.

[4] P. K. Varshney, Distributed Detection and Data Fusion. New York: Springer, 1997.

[5] P. K. Willett, P. F. Swaszek, and R. S. Blum, "The good, bad, and ugly: Distributed detection of a known signal in dependent Gaussian noise," IEEE Trans. Signal Process., vol. 48, no. 12, pp. 3266-3279, Dec. 2000.

[6] E. Drakopoulos and C. C. Lee, "Optimum multisensor fusion of correlated local decisions," IEEE Trans. Aerosp. Electron. Syst., vol. 27, no. 4, pp. 593-605, Jul. 1991.

[7] M. Kam, W. Chang, and Q. Zhu, "Hardware complexity of binary distributed detection systems with isolated local Bayesian detectors," IEEE Trans. Syst., Man, Cybern., vol. 21, no. 3, pp. 565-571, May/Jun. 1991.

[8] M. Kam, Q. Zhu, and W. S. Gray, "Optimal data fusion of correlated local decisions in multiple sensor detection systems," IEEE Trans. Aerosp. Electron. Syst., vol. 28, no. 3, pp. 916-920, Jul. 1992.

[9] C. Rago, P. K. Willett, and Y. Bar-Shalom, "Censoring sensors: A low-communication-rate scheme for distributed detection," IEEE Trans. Aerosp. Electron. Syst., vol. 32, no. 2, pp. 554-568, Apr. 1996.

[10] F. Gini, F. Lombardini, and L. Verrazzani, "Decentralised detection stratigies under communication constraints," in Proc. Inst. Elect. Eng., Part F: Radar, Sonar, Navigation, vol. 145, Aug. 1998, pp. 199-208.

[11] C. T. Yu and P. K. Varshney, "Paradigm for distributed detection under communication constraints," Opt. Eng., vol. 37, no. 2, pp. 417-426, Feb. 1998

[12] - "Bit allocation for discrete signal detection," IEEE Trans. Commun., vol. 46, pp. 173-175, Feb. 1998.

[13] T. Kasetkasem and P. K. Varshney, "Communication structure planning for multisensor detection schemes," in Proc. Inst. Elect. Eng., Part F: Radar, Sonar, and Navigation, vol. 148, Feb. 2001, pp. 2-8.

[14] J. Hu and R. Blum, "On the optimality of finite-level quantization for distributed signal detection,” IEEE Trans. Inf. Theory, vol. 47, no. 4, pp. 1665-1671, May 2001.

[15] J. Chamberland and V. V.Veeravalli, "Decentralized detection in sensor networks," IEEE Trans. Signal Process., vol. 51, no. 2, pp. 407-416, Feb. 2003.

[16] S. C. A. Thomopoulos and L. Zhang, "Distributed decision fusion with networking delays and channel errors," Inf. Sci., vol. 66, pp. 91-118, Dec. 1992.

[17] B. Chen, R. Jiang, T. Kasetkasem, and P. K. Varshney, "Channel aware decision fusion in wireless sensor networks," IEEE Trans. Signal Process., vol. 52, no. 12, pp. 3454-3458, Dec. 2004.

[18] T. Rappaport, Wireless Communications-Principles and Practices. Upper Saddle River, NJ: Prentice-Hall, 1996.

[19] G. L. Stüber, Principles of Mobile Communication, 2nd ed. Boston, MA: Kluwer, 2001

[20] B. Picinbono, "On deflection as a performance criterion in detection," IEEE Trans. Aerosp. Electron. Syst., vol. 31, no. 3, pp. 1072-1081, Jul. 1995.

[21] S. M. Kay, Fundamentals of Statistical Signal Processing II: Detection Theory. Englewood Cliffs, NJ: Prentice-Hall, 1998.

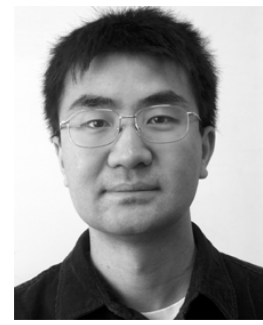

processing.

Dr. Niu received the Fusion 2004 Best Paper Award, in the 7th International Conference on Information Fusion, Stockholm, Sweden, in June 2004.

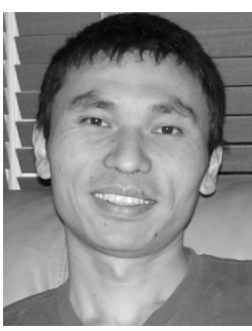

Biao Chen (S'97-M'99) received the M.S. degree in statistics and the Ph.D. degree in electrical engineering from the University of Connecticut, Storrs, in 1998 and 1999, respectively.

From 1999 to 2000, he was a Postdoctoral Research Associate with Cornell University, Ithaca, NY. Since 2000, he has been an Assistant Professor with the Department of Electrical Engineering and Computer Science, Syracuse University, Syracuse, NY. His area of interest mainly focuses on statistical signal processing with application to communication

and radar systems

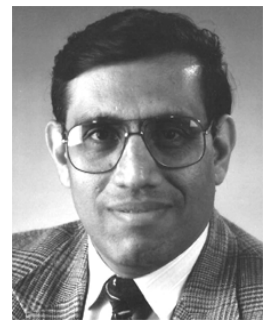

Pramod K. Varshney (F'97) was born in Allahabad, India, on July 1, 1952. He received the B.S. degree in electrical engineering and computer science (with highest honors), and the M.S. and Ph.D. degrees in electrical engineering from the University of Illinois at Urbana-Champaign in 1972, 1974, and 1976, respectively.

From 1972 to 1976 , he was a teaching and research assistant at the University of Illinois. Since 1976 , he has been with Syracuse University, Syracuse, NY, where he is currently a Professor of electrical engineering and computer science and the Research Director of the New York State Center for Advanced Technology in Computer Applications and Software Engineering. He served as the Associate Chair of the department from 1993 to 1996. He is also an Adjunct Professor of Radiology at Upstate Medical University in Syracuse, NY. His current research interests are in distributed sensor networks and data fusion, detection and estimation theory, wireless communications, image processing, radar signal processing, and remote sensing. He has published extensively. He is the author of Distributed Detection and Data Fusion (New York: Springer-Verlag, 1997). He has served as a consultant to several major companies.

Dr. Varshney was a James Scholar, a Bronze Tablet Senior, and a Fellow while at the University of Illinois. He is a member of Tau Beta Pi and is the recipient of the 1981 ASEE Dow Outstanding Young Faculty Award. He was elected Fellow of the IEEE in 1997 for his contributions in the area of distributed detection and data fusion. He was the Guest Editor of the special issue on data fusion of the PROCEEDINGS OF THE IEEE in January 1997. In 2000, he received the Third Millennium Medal from the IEEE and Chancellor's Citation for exceptional academic achievement at Syracuse University. He serves as a Distinguished Lecturer for the Aerospace and Electronic Systems (AES) society of the IEEE. He is on the editorial board of Information Fusion. He was the President of International Society of Information Fusion during 2001. 